\title{
COMPRENSIÓN DE TEXTOS EN CONTEXTOS DESFAVORECIDOS: EL EFECTO DE UN PROGRAMA DE INTERVENCIÓN EN LA HABILIDAD PARA DETECTAR ERRORES E INCONGRUENCIAS EN TEXTOS ESCRITOS*
}

\author{
READING COMPREHENSION IN DEPRIVED SOCIAL BACKGROUNDS: THE EFFECT OF \\ AN INTERVENTION PROGRAM BASED ON THE ABILITY TO DETECT ERRORS AND \\ INCONSISTENCIES IN WRITTEN TEXTS
}

\author{
Romina Cartoceti ${ }^{* *}$, Valeria Abusamra ${ }^{* * *}$, Rossana De Beni ${ }^{* * *}$ Y Cesare Cornoldi***
}

${ }^{*}$ Trabajo parcialmente financiado con una beca postdoctoral del Consejo Nacional de Investigaciones

Científicas y Técnicas (CONICET) otorgada a la primera autora.

**Doctora en Lingüística. Becaria Posdoctoral del Consejo Nacional de Investigaciones Científicas y Técnicas (CONICET). Jefe de Trabajos Prácticos de la Cátedra de Psicolingüística II de la Carrera de Letras de la Facultad de

Filosofía y Letras de la Universidad de Buenos Aires (UBA). E-Mail: romina.cartoceti@gmail.com

Avda. Díaz Vélez 4311, $7^{\circ}$ Piso, Dpto. A - (C1200AAK) Ciudad Autónoma de Buenos Aires.

***Doctora en Lingüística. Profesora Adjunta a cargo de la Cátedra de Psicolingüística II de la Carrera de Letras

de la Facultad de Filosofía y Letras de la Universidad de Buenos Aires (UBA).

****Licenciada y Profesora en Psicología. Profesora Regular de Psicología General y Psicología de la

Personalidad en la Carrera de Psicología y Directora del Laboratorio de Aprendizaje y Procesos Cognitivos de la Universidad de Pádova (Italia).

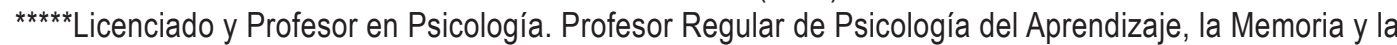

Cognición y de Fundamentos de la Psicología en la Universidad de Pádova (Italia).

Los autores agradecen la colaboración y el compromiso de los docentes, directivos y toda la comunidad educativa de la Escuela Creciendo Juntos de Paso del Rey (Provincia de Buenos Aires) para la administración de los instrumentos.

\section{RESUMEN}

La comprensión de textos es una de las competencias fundamentales para el desarrollo social $\mathrm{y}$ educativo de los seres humanos y constituye un tema esencial en la agenda educativa. Sin embargo, el estudio y la sistematización de los efectos de programas que buscan mejorar la comprensión lectora, sobre todo en el caso de alumnos pertenecientes a entornos sociales desfavorecidos, son todavía escasos. Se presentan los resultados de una intervención realizada en un grupo de alumnos de primero y segundo año de nivel medio y de contexto desfavorecido de una escuela de gestión social de la Provincia de Bue- nos Aires (Argentina). Dicha intervención estuvo basada en la habilidad metacognitiva de detectar errores e incongruencias, fundamental para el proceso de comprensión de un texto. Inicialmente fueron evaluados 50 adolescentes con un test de eficacia lectora, una prueba estandarizada de comprensión de textos y una prueba específica que evalúa la habilidad de detectar errores e incongruencias. Se conformó un grupo control con 23 participantes y un grupo experimental con los 27 restantes. El entrenamiento se llevó a cabo durante tres meses, con una frecuencia de una hora semanal y una vez finalizado, ambos grupos fueron reevaluados. Los resultados muestran un efecto positivo de la in- 
tervención sobre el grupo experimental, que alcanzó una diferencia estadísticamente significativa entre la cantidad de respuestas correctas de la evaluación inicial y de la evaluación final.

Palabras clave: Comprensión de textos; Modelo multicomponencial; Metacognición; Monitoreo; Adolescentes.

\section{ABSTRACT}

Reading comprehension is one of the core competencies for social and educational development of human beings and an essential topic in education agenda. However, the study and systematization of the effects of programs that seek to improve reading comprehension, especially in students from deprived social backgrounds, are still scarce. As a strategic skill that requires a conscious and active reader who can process the text and formulate a plan according to his or her purposes, reading comprehension must be flexible enough to adjust text demands. In this respect, a good reader should be aware of his or her cognitive abilities in order to understand a text successfully. Hence training in metacognitive skills improves significantly whole reading comprehension ability. Metacognitive training allows children to internalize and also anticipate the effective and useful strategies to be applied in each case.

This paper aims to promote social inclusion to adolescents from a deprived social environment. To gain this goal, a program was designed considering, measurable, agreed and communicable targets, according to real deadlines that fit the raised population needs and realities. It presents the results of an intervention in a group of students of first and second year from secondary school from a deprived context in Gran Buenos Aires (Argentina). This intervention was based on the ability to detect errors and inconsistencies, fundamental to understanding texts. 50 adolescents were initially evaluated with a reading efficiency test, a standardized reading comprehension test and a specific proof to assess the ability to detect errors and inconsistencies. 23 students were part of the control group and 27, were involved in the experimental group. The one hour training was held weekly for three months and once this training was completed, both groups were reevaluated. The training involved twelve sessions which kept the same structure. First the ability was orally introduced. A video, a song, a painting, language games, among other resources, were used to include an error (or more) and / or a mismatch (or more). Then, the students altogether discussed, orally, the strategies used to resolve de introduction activity in order to detect these errors and inconsistencies. In third place, an understanding activity that included a situation that supposed an error or inconsistency was introduced. A fourth step was to present a written activity to strengthen the target ability, asking the children to produce a similar situation. The session finally ended with a pooling of production. This scheme was repeated over the 12 sessions by modifying the content thereof. The last two activities were carried out in pairs to stimulate mutual learning. In every session the information was presented with Powerpoint Program, in differentiates place from, especially assigned for the interventions.

The results show a positive effect of the intervention on the experimental group, whose performance achieved a statistically significant difference between the number of correct responses from the initial to the second assessment. One-sample $t$ test was applied to compare the means of performance from the same population before and after the intervention. For the experimental group, statistically significant differences between means were checked before and after the intervention in both variables. time and right answers.

For the control group, which received no intervention but had traditional instruction, a $t$ test showed that the difference in performance between the first and second evaluation was not significant.

Key words: Reading comprehension; Multicomponent model; Metacognition; Monitoring; Adolescents.

\section{INTRODUCCIÓN}

La comprensión de textos es una habilidad cognitiva compleja e imprescindible para el desarrollo social y educativo de los seres humanos (Abusamra \& Joanette, 2012). Es por 
esto que se constituye en un tema de preocupación para los sistemas educativos dado que se encuentran ante la necesidad de generar estrategias y recursos idóneos que promuevan una instrucción satisfactoria.

\section{COMPRENSIÓN DE TEXTOS Y CONTEXTOS DESFAVO-} RECIDOS. UN ABORDAJE MULTICOMPONENCIAL

Hoy en día, el avance de los modelos teóricos aportados por distintas disciplinas y el desarrollo de instrumentos de evaluación específicos han permitido visualizar de manera más evidente el estado de la cuestión, colocando la temática en el centro del debate. Los resultados de las dos últimas ediciones de la prueba PISA (2009-2012) (OCDE, 2013) mostraron un descenso significativo en el rendimiento de los alumnos de países latinoamericanos, posicionándose muy por debajo del nivel esperado. Más allá de las controversias que generan estas pruebas, existe una percepción generalizada del incremento de las dificultades a la hora de comprender textos. El problema se pone especialmente de manifiesto en el caso de los alumnos insertos en contextos o entornos sociales desfavorecidos. Un trabajo de Ferreres, Abusamra y Squilacce (2010) demostró el impacto que la desigualdad de oportunidades educativas tiene sobre la comprensión lectora y las diferencias de rendimiento que acarrea. Otros trabajos también han corroborado dicho impacto (Abusamra, Cartoceti, Ferreres, Raiter, De Beni \& Cornoldi, 2014; Abusamra, Ferreres, Raiter, De Beni \& Cornoldi, 2010). Estos últimos remiten a la normatización del Test Leer para Comprender (Abusamra et al., 2010) realizada con una población de 2.094 niños de escuelas primarias de Capital Federal y Gran Buenos Aires, y del Test Leer para Comprender II (Abusamra, Cartoceti, Ferreres, Raiter, De Beni \& Cornoldi, 2014) que fue administrado a una población de 2.600 alumnos de escuelas secundarias de las mismas zonas. Los resultados evidenciaron diferencias en el rendimiento de acuerdo con las oportunidades educativas de las escuelas a las que asistían los alumnos. Dichas dife- rencias están relacionadas con las características socioeconómicas de la población escolar y las condiciones en las que se desarrollaba la actividad docente, independientemente de la gestión -privada o estatal- a la que dichas escuelas pertenecían. Otras investigaciones (Borzone, 1997; Cuadro \& Balbi, 2012; Diuk, 2003; Diuk \& Ferroni, 2014; Diuk, Signorini \& Borzone, 2000; Piacente, Marder \& Resches, 2006; Plana \& Fumagalli, 2013) demostraron la influencia que los factores ambientales tienen sobre las habilidades de alfabetización emergente e inicial. Todos los trabajos dieron cuenta empíricamente de que los alumnos provenientes de ambientes desfavorecidos presentaban un peor rendimiento en tareas que miden dichas habilidades. De allí entonces, la necesidad de tratamiento de la temática.

Comprender un texto implica generar una representación mental, un modelo de situación sobre lo que el texto trata (van Dijk \& Kinstch, 1983). Esto exige un lector activo que compute la información explícita e infiera lo que no está dicho a partir del conocimiento del mundo. Son muchas las teorías psicolingüísticas que han tratado de dar cuenta de este fenómeno (Kinstch, 1988; Kintsch \& van Dijk, 1978; Gernsbacher, 1990; van den Broek, Young \& Linderholm, 1999; van Dijk \& Kinstch, 1980, 1983). Pero, además de implicar complejos procesos cognitivos, la comprensión de un texto es una habilidad cultural que se ve fuertemente influenciada por las condiciones sociales y económicas en las que está inmerso el sujeto. En tanto habilidad cultural, la comprensión de textos puede y debe ser entrenada (Amado \& Borzone, 2011; Abusamra \& Joanette, 2012). Un estudio llevado a cabo por Meneghetti, Carretti, Abusamra, De Beni y Cornoldi (2009) ha verificado la eficacia que tiene un adecuado programa de intervención sobre la comprensión general.

El estudio que se informa consistió en la implementación de un programa de entrenamiento de la comprensión de textos a partir del trabajo tanto en habilidades cognitivas como metacognitivas, tomando como base una perspectiva multicomponencial (Abusamra et al., 2010; Abusamra, Casajús, Fe- 
rreres, Raiter, De Beni \& Cornoldi, 2014; De Beni, Cornoldi, Carretti \& Meneghetti, 2003). La idea que subyace a este modelo es que en el proceso de comprensión de un texto pueden identificarse subcomponentes necesarios para llevar adelante una comprensión exitosa. Se ha comprobado que la discriminación de componentes y el abordaje de cada uno en forma individual permiten una evaluación más certera y una intervención orientada y eficaz. El trabajo en una habilidad específica influye positivamente sobre las habilidades generales de comprensión lectora.

Según el modelo multicomponencial, son 11 las habilidades fundamentales que se ponen en marcha a la hora de comprender un texto. En primer lugar, el lector deberá identificar personajes, lugares y tiempos de una acción narrada, reconocer los hechos y secuencias y abordar aspectos ligados a la semántica léxica. Sin embargo, la comprensión lectora no se limita al procesamiento de palabras aisladas. Un lector competente debe ser capaz de detectar estructuras sintácticas, asignar el significado de las frases, resolver lazos de cohesión y generar inferencias. Además, es necesario que el lector sea capaz de establecer la jerarquía del texto para discriminar entre información relevante e irrelevante y a partir de esto construir un modelo mental sobre la base de la integración de la información textual y el conocimiento de mundo del lector. Por último, son determinantes las habilidades metacognitivas que permitan reflexionar sobre el propio proceso de comprensión. Para esto se requiere una buena habilidad de intuición (que permita que el lector anticipe las características del texto), de flexibilidad mental para adaptarse a las demandas de la tarea y de monitoreo para poder detectar errores e incongruencias (ver Figura 1).

\section{METACOGNICIÓN Y COMPRENSIÓN DE TEXTOS}

Definir metacognición no es una tarea fácil. Algunos investigadores consideran que la metacognición implica un conocimiento consciente y acciones deliberadas; mientras que para otros, la metacognición puede ser un proceso tácito y automático. Del mismo modo, hay quienes sostienen que la metacognición involucra emoción y motivación, mientras que otros prefieren conceptualizarla como conocimiento deslindado del afecto (Jacobs \& Paris, 1987). En línea con estos últimos autores, se puede pensar que la metacognición supone el conocimiento o el grado de conciencia acerca de los propios estados o procesos cognitivos (Jacobs \& Paris, 1987). Esta definición supone por un lado, considerar la conciencia metacognitiva en tanto conjunto de conocimientos que una persona tiene acerca del funcionamiento de la mente, y por otro, los procesos metacognitivos de control, que son el conjunto de mecanismos que rigen al proceso cognitivo durante su ejecución. En este sentido y como indican Jacobs y Paris (1987), el estudio de la metacognición en relación con la comprensión permite abordar la reflexión tanto sobre aspectos cognitivos como las estrategias empleadas, como así también sobre la autorregulación de los procesos de los propios comprendedores. Por lo tanto, estimular el trabajo a partir de estas habilidades se vislumbra como un campo promisorio para dar respuesta a las dificultades actuales de comprensión. Estas habilidades de reflexión de la que hablan los autores pueden proyectarse sobre tres categorías que refieren como conocimiento declarativo, procedural y condicional.

El conocimiento declarativo atañe a lo que se conoce de manera proposicional. Por ejemplo, un sujeto puede saber que conocer previamente el tema (familiaridad) sobre el cual tratará un texto, facilitará su comprensión. El conocimiento procedural, por su parte, implica la conciencia que las personas tienen sobre el proceso de pensamiento. Esto se refiere por ejemplo al conocimiento que un comprendedor tiene sobre los procesos cognitivos involucrados en determinada tarea: por ejemplo cómo resumir, cómo seleccionar ideas principales, etc. Por último, el conocimiento condicional supone la conciencia que el sujeto tiene sobre las condiciones que facilitan el aprendizaje. Por ejemplo, qué estrategias son efectivas frente a determinada tarea y cuándo emplearlas. 
Por otra parte, los estudios fundacionales de Flavell (1971) sobre metamemoria y posteriormente, los de Brown, Campione y Day (1981) han abierto una nueva perspectiva en las investigaciones sobre comprensión de textos. Brown (1981) y Brown, Armbruster y Baker (1986) distinguen entre los conocimientos relativos al texto -el objetivo de la lectura, las estrategias- y los relativos al sujeto como lector, como aspectos metacognitivos que deben ser considerados en la comprensión de un texto.

Entonces, como estrategia de intervención, propiciar el desarrollo de habilidades metacognitivas permitirá al lector / comprendedor adquirir un carácter más intuitivo sobre aspectos críticos del texto tales como los errores, las incoherencias y las ambigüedades, pudiendo organizar y planificar sus propios procesos para alcanzar una comprensión exitosa (Abusamra, Casajús, Ferreres, Raiter, De Beni \& Cornoldi, 2014). El valor de trabajar con una habilidad metacognitiva radica en el desarrollo de una conciencia particular por parte del lector / comprendedor sobre el propio proceso de comprensión de un texto.

Existen numerosos estudios que vinculan la habilidad de detección de errores con redes neurales explícitas que sostienen dicha actividad (Fleming \& Dolan, 2012; Miltner, Brown \& Coles, 1997). En este sentido es una habilidad que va evolucionando junto con el desarrollo del niño. Desde los inicios de los años ochenta varios estudios demostraron la relación que existe entre la habilidad para detectar errores e incongruencias y la habilidad de comprensión de textos. De hecho se ha demostrado que usualmente los malos comprendedores fallan, en comparación con la actuación de buenos, en la detección de errores e incongruencias (Garner \& Kraus, 1982; Jaramillo Arana, Montaña de la Cadena \& Rojas Reina, 2006; Ruffman, 1996; Vosniadou, Pearson \& Rogers, 1987) e incluyeron esta discusión dentro del paradigma de detección de errores (Winograd \& Johnston, 1982). Este es uno de los paradigmas que dominan el estudio de la investigación de las diferencias individuales en el monitoreo cognitivo
(Garner \& Kraus, 1982). Este monitoreo, sin embargo, entraña en sí un proceso metacognitivo: la detección de errores es parte del proceso de monitoreo de la lectura que permite al sujeto identificar que existe una inconsistencia o en el texto o en una parte del mismo, que le dificulta alcanzar una comprensión óptima del mismo. Para alcanzarla, el lector debe ser consciente de su propio proceso de comprensión, es decir, debe ser capaz de ir monitoreando on-line la construcción de su modelo mental del texto para poder detectar en qué momento éste entra en contradicción con la información proveniente del texto. Este último proceso, que entraña un carácter metacognitivo, resulta natural en un lector eficiente, pero representa una complicación extra para un lector que experimente dificultades. En este sentido, indagar sobre esta capacidad y entrenarla, debería redundar en una mejora en el proceso de comprensión.

En síntesis, la metacognición puede pensarse como un macroproceso; supone la capacidad de conciencia y de control voluntario para gestionar todos los procesos cognitivos, desde los simples a los complejos. Aplicado a la comprensión de un texto, implica tener conciencia y poder reflexionar sobre las propias habilidades del lector / comprendedor. Leer implica, fundamentalmente, comprender; sin embargo, un lector experto debe ser capaz de advertir cuando su comprensión no es adecuada, cuando algo le resulta confuso $\mathrm{o}$ es incongruente o erróneo en el texto y comporta un quiebre en su propio proceso de comprensión. Una adecuada capacidad para detectar errores o incongruencias presentes en un texto es un antecedente apropiado de una correcta habilidad de monitoreo (Cornoldi \& Oakhill, 1996). Por ese motivo, trabajar a partir de un texto que presente errores es una posibilidad de acceso para evaluar esta capacidad en cuestión.

Por este motivo, esta investigación se propuso trabajar con la habilidad para detectar errores e incongruencias a fin de poder entrenar la capacidad de monitoreo necesaria para descubrir incoherencias en algunas situaciones presentadas e interpretar de modo diferente una misma situación. La detección 
de errores supone considerar aquellas omisiones o incorrecciones referidas a los niveles fonológico y morfosintáctico. En cambio, la referencia a una incongruencia remite a incoherencias o equivocaciones en los niveles semántico y pragmático (Abusamra, Casajús, Ferreres, Raiter, De Beni \& Cornoldi, 2014).

\section{HIPÓTESIS Y OBJETIVOS}

Se estableció como hipótesis de base que la intervención en una o varias de las habilidades consideradas como fundamentales para llevar adelante una comprensión exitosa redundará en beneficio de la comprensión en general. De manera específica, subyace la hipótesis de que un entrenamiento sistemático en una habilidad metacognitiva de monitoreo mejorará el rendimiento del grupo experimental de manera significativa.

El objetivo de este estudio fue verificar el efecto de un programa de intervención aplicado a alumnos de una escuela que cuenta con un entorno social desfavorecido. Para este trabajo en particular se entrenó, tomando como base el modelo de múltiples componentes previamente explicado (Abusamra et al., 2010; Abusamra, Cartoceti, Ferreres, Raiter, De Beni \& Cornoldi, 2014), la habilidad para detectar errores e incongruencias, habilidad que refleja una capacidad metacognitiva fundamental para monitorear el propio proceso de lectura.

Además, se pretendió promover la inclusión social de una población educativa perteneciente a un entorno social desfavorecido a partir del planteo de metas consensuadas, medibles, comunicables y con plazos de cumplimiento que se ajusten a sus realidades y necesidades. Esto se articula a partir de las siguientes tareas:

1.- Evaluación de la comprensión lectora de una población de adolescentes de escuela secundaria perteneciente a entornos sociales desfavorecidos, a partir de un instrumento que valora múltiples componentes.

2.- Implementación de un programa de mejoramiento de la comprensión lectora a partir del trabajo en el área de detección de errores e incongruencias.

3.- Verificación de la eficacia del programa de mejoramiento.

\section{MÉTODO \\ PARTICIPANTES}

Participaron 50 alumnos de primer y segundo año de una escuela secundaria de la localidad de Moreno, ubicada en el oeste del Gran Buenos Aires, pertenecientes a entornos sociales desfavorecidos ${ }^{1}$. El grupo de primer año se constituyó como grupo experimental (27 estudiantes con una edad promedio de 13.3 años, 12 varones y 15 mujeres) y el grupo de segundo año fue tomado como grupo control (23 estudiantes con una edad promedio de 14.3 años, 7 varones y $16 \mathrm{mu}$ jeres). Los grupos difieren en edad porque el colegio cuenta sólo con una división por curso y el número de alumnos por curso no era suficiente para dividirlo en dos grupos (experimental y control) y realizar un análisis estadístico consistente. Esta segmentación de la muestra permitió observar si el grupo experimental, de menor edad, una vez finalizada la intervención, alcanzaba al menos el nivel inicial del grupo control, que pertenecía al curso inmediatamente superior.

\section{Procedimiento}

Ambos grupos fueron evaluados inicialmente. Al grupo experimental se le aplicó el programa de intervención durante las horas de clase, mientras que durante ese mismo período, el grupo control recibió la instrucción tradicional. Finalizado el mismo, ambos grupos fueron evaluados nuevamente.

1 Se entiende por entorno social desfavorecido un conjunto de circunstancias que afectan u obstaculizan el normal desarrollo cognitivo, físico, emocional y/o social de las personas que viven en ambientes de pobreza cultural y/o material (Sánchez Palomino \& Villega, 1998). 


\section{EVALUACIÓN DEL PERFIL DE COMPETENCIA LECTORA Y COMPRENSIÓN DE TEXTOS}

Durante la primera semana del mes de agosto de 2014 se evaluó el nivel de eficacia lectora y de comprensión de textos. Se administró el TECLE (Cuadro, Costa, Trías \& Ponce de León, 2009; Ferreres, Abusamra, Casajús \& China, 2011) para evaluar la habilidad de lectura y comprensión de oraciones y dos textos screening de la comprensión (Abusamra et al., 2010; Abusamra, Cartoceti, Ferreres, Raiter, De Beni \& Cornoldi, 2014). Estas primeras pruebas se tomaron para descartar aquellos casos que rindieran por debajo de la media en la prueba de TECLE, cosa que no ocurrió. Y para determinar, a partir de los textos screening, un perfil de rendimiento en comprensión lectora que permitiera decidir qué habilidad trabajar durante la intervención. A partir de esta primera evaluación se decidió trabajar con el área 11 del Test Leer para Comprender II: Errores e Incongruencias (Abusamra, Cartoceti, Ferreres, Raiter, De Beni \& Cornoldi, 2014) que mide la habilidad de monitoreo.

\section{IMPLEMENTACIÓN DEL PROGRAMA DE ENTRENA- MIENTO}

Se diseñaron 12 sesiones de intervención que fueron aplicadas con una periodicidad semanal de una hora de duración cada una. Las mismas se desarrollaron durante los meses de agosto, septiembre y octubre de 2014. El esquema de entrenamiento se aplicó exclusivamente en el grupo experimental.

La Figura 2 sintetiza el procedimiento general que se siguió para la intervención. El mismo fue aplicado sistemáticamente en cada sesión. En el mes de noviembre de 2014 se realizó el re-test.

\section{INSTRUMENTOS}

Para evaluar eficacia lectora se administró el TECLE (Cuadro et al., 2009; Ferreres et al., 2011). Es un test que requiere habilidades de decodificación y comprensión de oraciones simples. Consta de 64 oraciones en las que falta la última palabra. Los sujetos deben elegir en cada caso la palabra que completa de modo correcto cada oración (sólo hay una entre cuatro opciones) y para eso cuenta con 5 minutos. Las opciones de respuesta incluyen: una correcta, una palabra que no completa adecuadamente la oración y dos pseudopalabras que guardan una relación formal (fonológica u ortográfica) con el blanco. Esta prueba se utilizó con el fin de detectar y excluir de la muestra final, aquellos casos que presentaran serias dificultades de decodificación, un proceso de bajo nivel fundamental para la adecuada comprensión del texto.

Para evaluar el nivel general de comprensión lectora, se utilizó un screening de comprensión de textos: un texto narrativo, seguido de 10 preguntas con modalidad multiple choice, tomado del Test Leer para Comprender II (Abusamra, Cartoceti, Ferreres, Raiter, De Beni \& Cornoldi, 2014), que evalúa la habilidad de comprensión lectora a partir de un modelo teórico multicomponencial. Supone el testeo de 11 habilidades (ver su descripción en el apartado Introducción) y fue normatizado con 2.850 niños de Capital Federal y Gran Buenos Aires de 13 a 15 años de edad. El test incluye dos textos screening de la comprensión, sus preguntas evalúan las habilidades consideradas como fundamentales por el modelo multicomponencial de la evaluación de la comprensión lectora (ver Figura 1). A partir de analizar los errores cometidos por los alumnos en la resolución de las mismas se decidió trabajar con el área 11 de Errores e Incongruencias del Test Leer para Comprender II. Esta decisión estuvo fundada en el recuento y comparación de los errores cometidos en las respuestas a las preguntas de los screenings, viéndose afectadas en mayor porcentaje aquellas que remiten a una habilidad metacognitiva. A partir del estudio normativo realizado en el Test Leer para Comprender II puede verse cómo la habilidad de detección de errores e incongruencias es la que más dificultades supuso para su resolución en los alumnos. De acuerdo con las normas de este test, todos los alumnos de to- 
das las escuelas incluidas rindieron aproximadamente 2 puntos menos en el promedio de respuestas correctas con respecto al rendimiento de los mismos grupos en las otras áreas (Abusamra, Cartoceti, Ferreres, Raiter, De Beni \& Cornoldi, 2014).

Para la evaluación del área específica a entrenar, se utilizó el área 11 del Test Leer para Comprender II: Errores e Incongruencias (Abusamra, Cartoceti, Ferreres, Raiter, De Beni \& Cornoldi, 2014). En este caso, se presentó una serie de fragmentos textuales, seguidos de preguntas (10 en total) con cuatro opciones de respuestas, de las cuales sólo una era la correcta. De acuerdo con las instrucciones del test, los alumnos recibieron el material en un cuadernillo y se les pidió que procedieran a la lectura y respuestas de las mismas lo más rápido posible, pero de manera segura. Con el objetivo de evaluar también la eficiencia y la velocidad para hacer la tarea, se registró el tiempo que medió entre el inicio de la prueba y la devolución del cuadernillo completo por parte de los alumnos.

Para las intervenciones en el grupo experimental, se elaboró material acorde respetando el esquema de entrenamiento antes planteado (ver Figura 2). Parte de estos materiales fueron extraídos del Programa Leer para Comprender II (Abusamra, Casajús, Ferreres, Raiter, De Beni \& Cornoldi, 2014). Las 12 sesiones guardaron siempre la misma estructura: en primer lugar se comenzaba por introducir oralmente la habilidad en cuestión. En general, el material que se utilizó para esta instancia fue un video, una canción, algún material pictórico de artistas plásticos, juegos lingüísticos, entre otros recursos, que incluyeran un error (o varios) y/o una incongruencia (o varias). Luego, se trabajaban también grupal y oralmente, las estrategias puestas en juego para poder detectar dichos errores e incongruencias. A continuación, se planteaba una actividad de comprensión de una situación que presentara un error o una incongruencia y luego, se afianzó la habilidad pidiendo la producción de una situación similar. La sesión cerraba, finalmente, con una puesta en común de las producciones. Este esquema se repitió a lo largo de las doce sesio- nes modificándose el contenido de las mismas. Las últimas dos actividades se realizaron en pares para estimular la enseñanza recíproca. En todas las sesiones se trabajó con un PowerPoint en un espacio de la escuela diferente al del aula y destinado especialmente para las intervenciones.

Finalmente, ambos grupos fueron nuevamente evaluados con el área 11 de Errores e Incongruencias del Test Leer para Comprender II.

\section{Resultados}

Con el objetivo de caracterizar los rendimientos antes y después de la intervención se calcularon las medias de rendimiento y sus dispersiones. Se aislaron las siguientes variables: cantidad de respuestas correctas en el área 11 antes y después de la intervención y tiempo demorado en la resolución del área antes y después de la intervención. La Tabla 1 presenta las medias y los desvíos estándar obtenidos por ambos grupos en las dos instancias de administración. Luego se aplicó una prueba $t$ para muestras relacionadas para comparar las medias de rendimiento de la misma población antes y después de la intervención. En el caso del grupo experimental se verificaron diferencias estadísticamente significativas entre las medias antes y después de la intervención tanto en la variable de respuestas correctas (A11 antes / A11 después), $(t=-2.543 ; g l=26, p<.05)$; como en la variable tiempo (Tpo antes / Tpo después), $(t=8.054 ; g l=26, p<.001)$. De hecho, la media de rendimiento en el índice de respuestas correctas aumentó de $4.41(D E=2.34)$ a 5.22 $(D E=2.08)$ y la media de rendimiento en la variable tiempo disminuyó de $24096 \mathrm{msg}$ $(D E=7533.15)$ a $18570 \mathrm{msg}(D E=4542.17)$ (ver Tabla 2).

En el caso del grupo control, que no recibió la intervención sino que tuvo la instrucción tradicional, una prueba $t$ mostró que la diferencia de rendimiento entre la primera y la segunda evaluación no fue significativa $(t=-.454 ; g l=22, p>.05)$. Aunque sí lo fue en relación con el tiempo $(t=10.127 ; g l=22$, 
$p<.001)$. El puntaje en el test de comprensión lectora se mantuvo substancialmente similar, con un cambio menor y no significativo $(p=.65)$, de 6.43 respuestas correctas. Esto demuestra que la mejoría observada en el grupo experimental no se explica como un efecto de la re-evaluación.

Como se ha podido comprobar, sólo la cantidad de respuestas correctas mostró una diferencia estadísticamente significativa en el grupo experimental, exclusivamente. La variable tiempo mostró una diferencia significativa en ambos grupos. Esto puede deberse a que el tiempo refleja, no sólo la eficacia de la intervención en el caso del grupo experimental, sino que también es un valor que representa la familiarización con el tipo de tarea. De este modo, la primera exposición a la tarea puede resultar menos habitual para los alumnos. En cambio, en la segunda exposición ya conocen la metodología de trabajo, lo que se podría traducir en una disminución importante del tiempo en el caso de los dos grupos. Sin embargo, esta mejoría en el tiempo, no se vio acompañada de una mejoría significativa en el rendimiento en la tarea de comprensión ya que la cantidad de respuestas correctas no aumentó significativamente.

Por último, es necesario observar que el grupo experimental no alcanzó, luego de la intervención, el nivel basal en la variable cantidad de respuestas correctas del grupo control que era de mayor edad. El grupo experimental inició este estudio con 4.40 respuestas correctas y alcanzó un total de 5.22 respuestas correctas al finalizar la intervención, mientras que el grupo control inició este estudio con un rendimiento de 6.21 respuestas correctas y lo culminó con 6.44 respuestas correctas. Esto, lejos de debilitar el efecto de la intervención, lo resalta porque el grupo control, que inició el trabajo con una diferencia de casi más de 2 puntos con respecto al grupo experimental, terminó con una diferencia cercana a 1 punto y no logró mejorar significativamente su propio rendimiento. Para probar esto se realizó una prueba $t$ para muestras independientes para comparar, esta vez, las medias de rendimiento de ambos grupos (control y experi- mental) antes y después de la intervención. Los resultados mostraron que existía una diferencia estadísticamente significativa entre los rendimientos iniciales $(t=-2.697 ; \mathrm{gl}$ : 48 , $p<.05$ ), pero esta diferencia no se mantuvo significativa en la segunda evaluación $(t=-1.768 ; g l: 48, p>.05$, n.s.). Una vez más se confirma la efectividad de la intervención.

\section{Discusión}

La comprensión de textos es una habilidad estratégica que exige un lector consciente y activo que pueda formular un plan de lectura de acuerdo a sus propósitos, lo suficientemente flexible como para ir ajustándolo a medida que avanza el procesamiento del texto. En este sentido un buen lector deberá tener conciencia y control sobre las actividades cognitivas que debe realizar para poder comprender exitosamente un texto. De aquí que el entrenamiento en habilidades metacognitivas colabore significativamente con la mejoría de la comprensión lectora en general. Este tipo de entrenamiento, combinado con el trabajo cognitivo, permite que los niños interioricen las estrategias que utilizan y anticipen, además, el conocimiento de cuáles son eficaces y cuándo es útil aplicarlas.

La relación de necesidad que vincula la comprensión de textos con la metacognición radica en el poder reflexivo del constructo. Esta última permite poner el foco de reflexión en aspectos como el plan de lectura, el monitoreo del proceso de comprensión que permite detectar fallas en dicho proceso y compensarlas on-line, aspectos todos necesarios para lograr una comprensión exitosa. La metacognición enfatiza la participación activa del lector (Jacobs \& Paris, 1987) que debe involucrarse en el análisis de la tarea y en la estrategia que empleará. Más allá de la complejidad del constructo de metacognición, existe un aparente acuerdo en cuanto a lo que significa la habilidad en sí misma. Sin embargo, todavía continúa siendo un tema de debate cuál es el método más eficaz para intervenir y mejorar la comprensión lectora a 
partir de esta perspectiva metacognitiva (cf., MacKeown \& Beck, 2009; Williams \& Grant Atkins, 2009). Incluso se ha discutido mucho el verdadero efecto de las intervenciones basadas en metacognición bajo la crítica de que se enseña al niño más sobre qué es la lectura que acerca de cómo leer (y comprender) adecuadamente. Pero lo cierto es que, cuando se han podido medir adecuadamente los rendimientos antes y después de las intervenciones, los resultados han sido alentadores.

El paradigma de detección de errores supone un punto de partida relevante para el entrenamiento de una conciencia metacognitiva del proceso de comprensión. Trabajar a partir de la detección y corrección de errores estimula la habilidad de autorregulación y de reflexión sobre la capacidad o competencia de lectura alcanzada y las demandas cognitivas en sí mismas, constituyéndose en indicadores de los factores que influyen en la habilidad para capturar el proceso de comprensión. Este trabajo se ha propuesto evaluar el efecto de un programa de intervención en comprensión de textos en un grupo de alumnos de entornos sociales desfavorecidos que asiste a una escuela del Gran Buenos Aires. Para esto se seleccionaron dos grupos, uno experimental y otro control que fueron evaluados con los mismos instrumentos en una instancia previa y una posterior a la intervención. Sólo el grupo experimental recibió un entrenamiento sistemático que supuso el trabajo desde una perspectiva tanto cognitiva como metacognitiva. El programa de intervención consistió en 12 sesiones de una hora con una periodicidad semanal, luego de las cuales ambos grupos fueron reevaluados. Como se destacó en Resultados, sólo el grupo experimental demostró diferencias significativas entre las evaluaciones previas y posteriores a la intervención. Se ha intervenido sobre una habilidad puntual, la detección de errores e incongruencias. Un lector experto es capaz de detectar cualquier error o incongruencia que plantee el texto y que obstaculice la comprensión del mismo, incluso, muchas veces los corrige automáticamente durante la lectura (Abusamra, Cartoceti et al., 2014). De hecho, una habilidad de este tipo es predictora de una buena habilidad de automonitoreo (Cornoldi \& Oakhill, 1996). El trabajo sistemático a través de las doce intervenciones potenció la capacidad de los alumnos para transferir las estrategias que iban surgiendo en la práctica y generalizarlas a otras intervenciones así como a otros contextos de trabajo.

Como se ha dicho previamente, la comprensión de textos es una habilidad cultural que puede entrenarse y mejorarse. Es una conducta compleja y propia de la especie humana y representa, sin lugar a dudas, una competencia fundamental para el aprendizaje y el desarrollo tanto cultural como social del sujeto. En este sentido debe convertirse en uno de los temas prioritarios de las agendas educativas de todos los países. Los niños de entornos sociales desfavorecidos y bajas

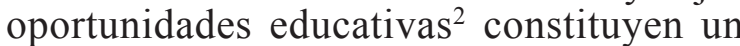
grupo en franca desventaja. Trabajar con poblaciones de entornos sociales desfavorecidos resulta, ni más ni menos, que en la propia inclusión social. El programa de intervención diseñado mostró ser un instrumento eficaz para trabajar la comprensión lectora. Pero además, es importante señalar que el trabajo sistemático para que los alumnos monitoreen sus propias estrategias de abordaje de los textos y para que puedan hacer un uso efectivo de las mismas es factible de realizarse en articulación con la instrucción tradicional. Y lo que es más importante, trabajar desde un abordaje tanto cognitivo como metacognitivo redunda en una mejoría para

2 Se consideran escuelas de bajas oportunidades educativas a aquellas que cumplen con los siguientes criterios: (1) la matrícula está conformada por alumnos provenientes de un nivel socioeconómico bajo; (2) provee merienda reforzada a los alumnos; (3) tiene un 30\% de alumnos repetidores y una tasa de ausentismo y deserción escolar superior al 14\%; (4) equipamiento básico o deficiente; (5) jornada simple (por incapacidad de recursos para implementar la jornada completa); (6) escasa o ninguna actividad extracurricular (Abusamra et al., 2010). 
la habilidad general de la comprensión de textos.

Es un desafío a futuro replicar este tipo de trabajos en otros grupos etarios y también en otros países porque de este modo estaremos generando más posibilidades para los grupos sociales más desamparados y se los estará compensando en término de oportunidades.

FIGURA 1

ESQUEMA DEL MODELO DE MÚLTIPLES COMPONENTES PARA EVALUAR LA COMPRENSIÓN DE TEXTOS

(ABUSAMRA ET AL., 2010)

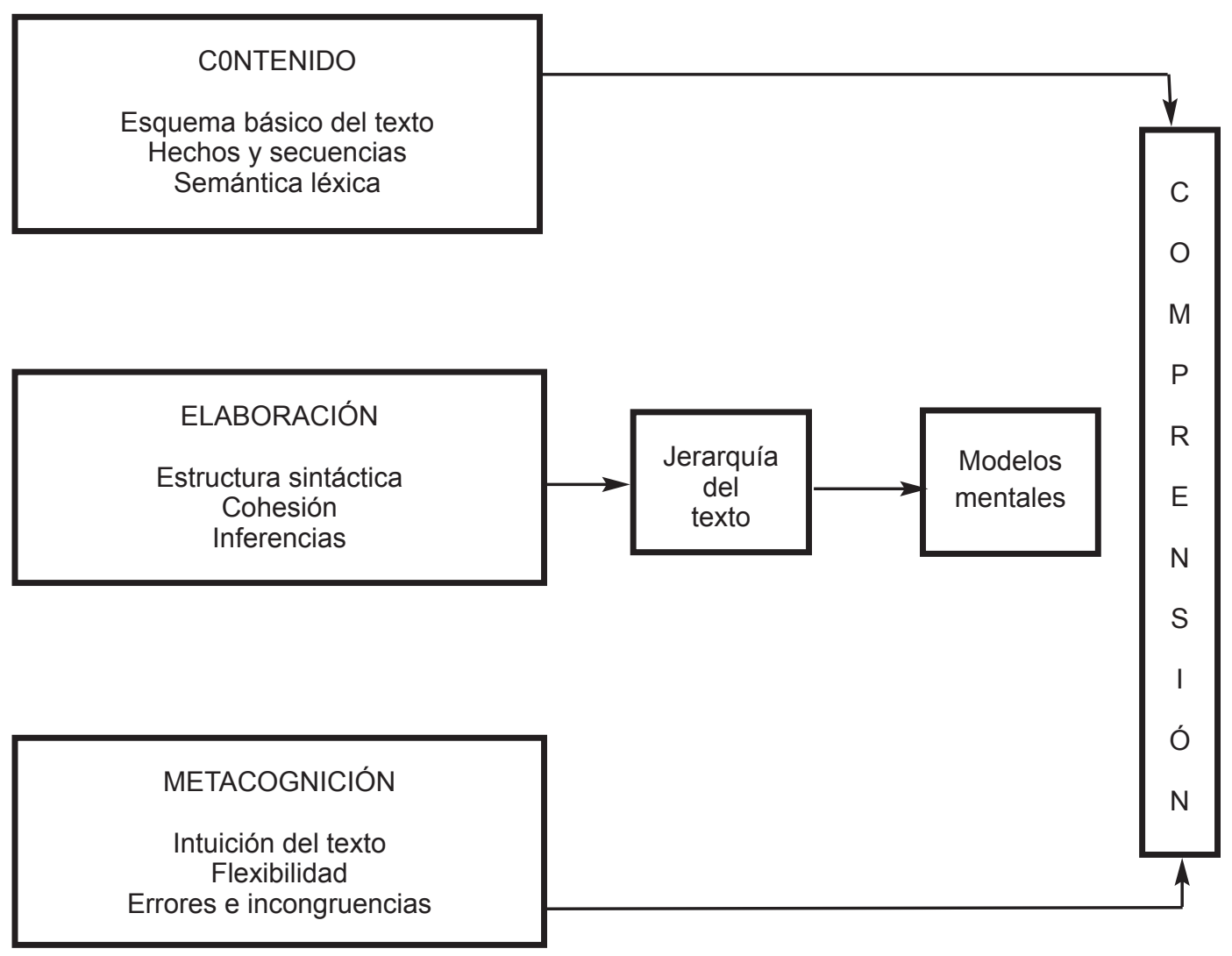


FIGURA 2

Procedimiento DE APLICACIÓN DEL PROGRAMA

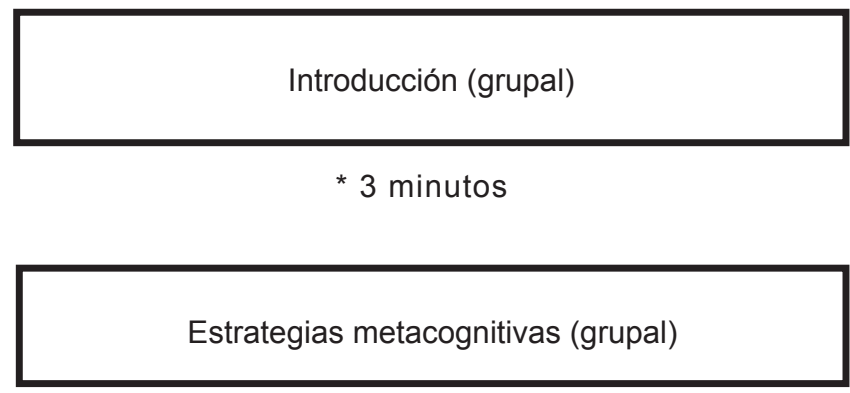

* 12 minutos

Comprensión: enseñanza recíproca (en parejas)

* 15 minutos

Producción (en parejas)

* 15 minutos

Puesta en común (grupal)

* 10 minutos

\section{TABLA 1}

ESTADÍSTICOS DESCRIPTIVOS DE MUESTRAS RELACIONADAS: MEDIAS Y DESVÍOS ESTÁNDARES PARA EL GRUPO EXPERIMENTAL

\begin{tabular}{|c|c|c|c|c|}
\hline & $M$ & $N$ & $D E$ & Error típ. de la media \\
\hline Par1 A11 Antes & 4.4074 & 27 & 2.34126 & .45058 \\
A11 Dsp & 5.2222 & 27 & 2.08167 & .40062 \\
& & & 4542.33681 & 874.17313 \\
\hline
\end{tabular}




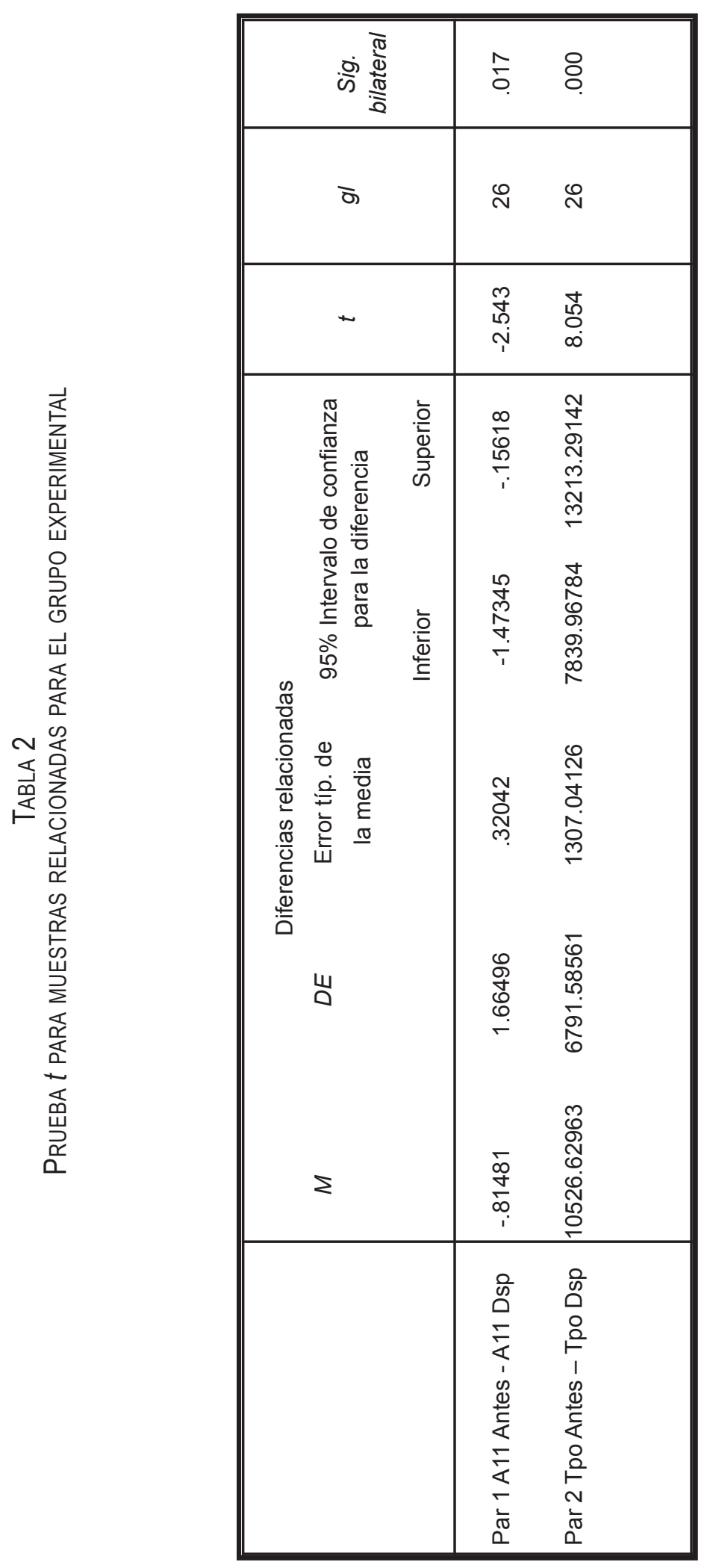




\section{ANEXo 1}

\section{EJEMPLO DE UNA INTERVENCIÓN MODELO}

Teniendo en cuenta el procedimiento presentado en la Figura 2, una posible intervención en detección de errores e incongruencias podría considerar las siguientes actividades:

1.- Introducción (3 minutos aproximadamente): Presentar instrucciones o imágenes que contengan algún error 0 incongruencia. Por ejemplo:

(1) En un jarabe contra la tos para niños dice en su prospecto:

- Este medicamento puede causar somnoliencia. No conduzca automóviles ni maneje maquinaria pesada después de consumirlo.

(2) En una tira de luces de Navidad fabricadas en China:

Para usar SOLO en el interior o en el exterior.

(3) En un paquete de frutos secos de American Airlines:

- Instrucciones: abrir el paquete. Comer los frutos secos

(4) En una sierra eléctrica sueca se da la siguiente instrucción:

- No intente detener la sierra con las manos o las piernas.

(5) En la caja de un disfraz infantil de "Superman":

-Llevar este disfraz puesto no le permite al niño la capacidad de volar.

Estrategias metacognitivas (12 minutos). Luego se trabaja con los alumnos sobre los aspectos de estas instrucciones: los errores, lo absurdo, las incongruencias y contradicciones que presentan, etc. Se conversa grupalmente sobre cómo detectaron esas incongruencias, qué información entra en contradicción con el propio conocimiento de mundo, por ejemplo en el caso del prospecto del jarabe para niños; o qué información entra en contradicción con otra información del mismo instructivo, por ejemplo en el caso de las luces de Navidad.

Comprensión (enseñanza recíproca: 15 minutos). En parejas, se les pide a los alumnos que resuelvan la siguiente actividad. Se lee solo la consigna de modo grupal a fin de asegurar la comprensión de la misma. 
Normas de convivencia para la escuela secundaria

Leer las siguientes normas y corregir los errores para que la convivencia pueda cumplirse efectivamente. En cada norma aparece una incongruencia.

\section{Acerca de la inasistencia}

Se computará un cuarto de falta cuando el alumno llegue con un retraso de hasta 10 minutos en la primera hora de clase y media falta cuando el alumno llegue con un retraso menor a 10 minutos.

Cuando el $4 \%$ de los alumnos de un mismo curso se encuentre ausente sin justificación, se considerará falta colectiva, computándose el doble de la inasistencia correspondiente al turno.

La inasistencia a un examen o evaluación por enfermedad debe ser comunicada y justificada con certificado médico antes de la misma para tener derecho a una nueva mesa de examen.

Acerca de la vestimenta y consideraciones generales

"Los varones y niñas asistirán con pantalón tipo jeans, remera blanca con mangas y zapatos o zapatillas deportivas. A las clases de educación física asistirán con equipo deportivo. NO se permitrá bajo ninguna circunstancia el uso de jogging o pantalones de gimnasia.

No está permitido el uso de celular, MP3, MP4, etc. Salvo en el caso de examen.

Está terminantemente prohibido el uso de alhajas. Sólo se permitirá llevar anillos si se portan en los dedos." (Abusamra, Casajús, Ferreres, Raiter, De Beni \& Cornoldi, 2014, p.165. Fragmento).

PRODUCCIÓN (15 minutos). Con la misma pareja se les pide a los alumnos que escriban un texto instructivo para un producto que elijan que tenga varias ideas ridículas y absurdas.

PUESTA EN COMÚN (10 minutos). Se finaliza la intervención con la exposición de los alumnos del trabajo de comprensión y producción. En ambos casos se vuelve a reflexionar sobre las estrategias puestas en juego en ambas tareas. 


\section{REFERENCIAS BIBLIOGRÁFICAS}

Abusamra, V., Cartoceti, R., Ferreres, A., Raiter, A., De Beni, R. \& Cornoldi, C. (2014). Test Leer para Comprender II. Evaluación de la comprensión de textos [Read to understand Test II. Reading comprehension assessment]. Buenos Aires: Paidós.

Abusamra, V., Casajús, A., Ferreres, A., Raiter, A., De Beni, R. \& Cornoldi, C. (2014). Programa Leer para Comprender II [Read to understand program II]. Buenos Aires: Paidós.

Abusamra, V., Ferreres, A., Raiter, A., De Beni, R. \& Cornoldi, C. (2010). Test Leer para Comprender. Evaluación de la comprensión de textos [Read to understand Test. Reading comprehension assessment]. Buenos Aires: Paidós.

Abusamra, V. \& Joanette, Y. (2012). Lectura, escritura y comprensión de textos: Aspectos cognitivos de una habilidad cultural [Reading, writing and reading comprehension: Cognitive aspects of a cultural skill]. Neuropsicologia Latinoamericana, 4(1), i-iv.

Amado, B. \& Borzone, A.M. (2011). La comprensión de textos expositivos: Relevancia del conocimiento previo en niños de distintos entornos socioculturales [Understanding expository texts: relevance of prior knowledge in children from different social environments]. Interdisciplinaria, 28(2), 261-277.

Borzone, A.M. (1997). El proceso de alfabetización en niños pequeños: Diferencias socio-culturales [Literacy in young children: Sociocultural differences]. Tesis doctoral no publicada. UBA. Buenos Aires.

Brown, A.L. (1981). Metacognition: The development of selective attention strategies for learning from texts. En M.L. Kamil (Ed.), Directions in reading: Research and instruction (pp. 21-43). Washington DC: National Reading Conference.

Brown, A.L., Armbruster, B.B. \& Baker, L. (1986). The rol of metacognition in reading and studying. En J. Orasanu (Ed.), Reading com- prehension: From research to practice. (pp. 4975). Hillsdale: LEA.

Brown, A.L., Campione, J.C. \& Day, J.D. (1981). Learning to learn: On training students to learn from texts. Educational Researcher, 10, 14-21.

Cornoldi, C. \& Oakhill, J. (1996). Reading comprehension difficulties. Mahwah, NJ: Erlbaum.

Cuadro, A. \& Balbi, A. (2012). Las diferencias socioeconómicas y la lectura: Algunas claves para analizar los resultados de las evaluaciones PISA [Socioeconomic differences and reading: Some keys to analyze the results of PISA assessments]. Revista de Neuropsicología Latinoamericana, 4(1), 57-64. http://dx.doi.org/10 5579/rnl2012.0093

Cuadro, A., Costa, D., Trías, D., \& Ponce de León, P. (2009). Evaluación del Nivel Lector. Manual técnico del Test de Eficacia Lectora de J. Marín y M. Carrillo [Reading level test: Technical manual of Efficiency Reading Test]. Montevideo: Prensa Médica Latinoamericana.

De Beni, R., Cornoldi, C., Carretti, B. \& Meneghetti, C. (2003). Nuova guida alla comprensione del testo [New guide to text comprehension]. Volumen 1. Trento: Erickson.

Diuk, B. (2003). Procesos de enseñanza y aprendizaje inicial de la lectura y la escritura en niños de sectores urbanos marginales [Inicial teaching and learning processess of reading and writing in children from marginal urban backgrounds]. Tesis doctoral no publicada. Facultad de Humanidades y Ciencias de la Universidad Nacional de La Plata (Argentina).

Diuk, B.G. \& Ferroni, M.V. (2014). Aprendizaje de letras en niños preescolares de nivel socioeconómico bajo [Letter learning in pre-school children growing in poverty]. Interdisciplinaria, 31(1), 25-37. http://dx.doi.org/10.16888/ interd.2014.31.1.2

Diuk, B., Signorini, A. \& Borzone, A.M. (2000). Las estrategias tempranas de lectura de palabras en niños de $1^{\circ}$ a $3^{\circ}$ año de educación general básica: Un estudio comparativo entre niños procedentes de distintos sectores sociales [The 
early word reading strategies in children from 1 st to 3rd year of basic education: A comparative study between children from different social background]. Psykhe, 12, 51-56.

Ferreres, A., Abusamra, V., Casajús, A. \& China, N. (2011). Adaptación y estudio preliminar de un test breve para evaluar la eficacia lectora (TECLE) [Adaptation and preliminary study of a short test to evaluate reading efficiency (TECLE)]. Revista Neuropsicología Latinoamericana, 3(1), 1-7. http.//dx.doi.org:105579/rn 12011.0040

Ferreres, A., Abusamra, V. \& Squilace, M. (2010). Comprensión de textos y oportunidades educativas [Reading comprehension and educational opportunities]. Actas del Congreso Iberoamericano de Educación. Metas 2021. Ciudad Autónoma de Buenos Aires. Argentina.

Flavell, J.H. (1971). First discussant's comments: What is memory development the development of? Human Development, 14, 272-278.

Fleming, S. \& Dolan, S. (2012). The neural basis of metacognitive ability. Philosophical Transaction of the Royal Society B: Biological Sciences, 367(1594), 1338-1349. http.//dox.doi.org:10.10 98/rstb.2011.0417

Garner, R. \& Kraus, C. (1982). Monitoring of understanding among seventh graders: An investigation of good comprehender-poor comprehender differences in knowing and regulating reading behaviours. Educational Research Quarterly, 6, 5-12. http.//dox.doi.org/ 10.1080//0270271820030102

Gernsbacher, M.A. (1990). Language comprehension as structure building. Hillsdale, NJ: Erlbaum.

Jacobs, J. \& Paris, S. (1987). Children's metacognition about reading: issues in definition, measurement and instruction. Educational Psychologist, 22(3-4), 255-278. http.//dox. doi.org: 10.1080/00461520.1987.9653052

Jaramillo Arana, A., Montaña de la Cadena, G. \& Rojas Reina, L. (2006). Detección de errores en el proceso metacognitivo de monitoreo de la comprensión lectora en niños [Error detection in metacognitive monitoring process of reading comprehension in children]. Revista Latinoamericana de Ciencias Sociales, Niñez y Juventud, 4(2), 4-17.

Kintsch, W. (1988). The role of knowledge in discourse comprehension: A construction-integration model. Psychological Review, 95(2), 163-182. http://dx.doi.org/10.1037/0033-295X. 95.2.163

Kintsch, W. \& van Dijk, T. (1978). Toward a model of text comprehension. Psychological Review, 85(5). http://dx.doi.org/10.1037/0033-295X.85. 5.363

MacKeown, M. \& Beck, (2009). The role of metacognition in reading comprehension. En D. Hacker, J. Dunlasky \& A. Graesser (Eds.), Handbook of metacognition in education (Cap. 1, pp. 7-25). Nueva York: Routledge.

Meneghetti, C., Carretti, B., Abusamra, V., De Beni, R. \& Cornoldi, C. (2009). El mejoramiento de la comprensión de textos desde una perspectiva componencial [Improving reading comprehension from a componential perspective]. Revista Ciencias Psicológicas, 3(2), 185- 192.

Miltner, W. Brown, Ch. \& Coles, M. (1997). EventRelated Brain Potentials Following Incorrect Feedback in a Time-Estimation Task: Evidence for a "generic" neural system for error detection. Journal of Cognitive Neuroscience, 9(6), 788-798. http.//dox.doi.org:10.1162/jocn.199 7.9.6.788

OCDE (2013). PISA 2012. Programa para la evaluación internacional de los alumnos. Informe español [PISA 2012 Programme for international student assessment. Spanish report]. Vol 1: Resultado y contexto. Ministerio de Educación, Cultura y Deporte Madrid.

Piacente, T., Marder, S. \& Resches, M. (2006). Condiciones de la familia y del niño para la alfabetización [Terms of family and child literacy]. Comisión de Investigaciones Científicas. La Plata. Gobierno de la Provincia de Buenos Aires. 
Plana, M.D. \& Fumagalli, J. (2013). Habilidades y conocimientos constitutivos de la alfabetización temprana: Semejanzas y diferencias según el entorno social y las oportunidades educativas [Early literacy skills: Similarities and differences caused by social environment and school opportunities]. Interdisciplinaria, 30(1), 5-24. http://dx.doi.org/10.16888/interd.2013.30.1.1

Ruffman, T. (1996). Reassessing children's comprehension-monitoring skills. En C. Cornoldi \& C. Oakhill (Comps.), Reading comprehension difficulties: Processes and intervention (Cap. 3, pp. 33-67). USA: Erlbaum.

Sánchez Palomino, A. \& Villegas, F. (1998). Dificultades por deprivación sociocultural. En A. Sánchez Palomino \& J.A. Torres González (Coords.), Educación especial II. Ámbitos específicos de intervención [Special education II. Specific areas of intervention] (pp. 161-183). Madrid: Pirámide.

van den Broek, P., Young, M. \& Linderholm, Y.T.T. (1999). The landscape model of reading: Inferences and the online construction of a memory representation. En H. van Oostendorp \& S. Goldman (Eds.), The construction of mental representations during reading (pp. 71-98). Nueva York: Psychology Press.

van Dijk, T.A. (1980). Estructuras y funciones del discurso [Structures and functions of discourse]. Buenos Aires: Siglo XXI.

vanDijk, T.A. \& Kintsch, W. (1983). Strategies of discourse comprehension. New York: Academic Press.

Vosniadou, S., Pearson, P. \& Rogers, T. (1987). What causes children's failure to detect inconsistencies in text. Center for the study of reading. Technical Reports $N^{\circ} 401$. Illinois: University of Illinois.

Williams, J. \& Grant Atkins, J. (2009). The role of metacognition in teaching reading comprehension in primary students. En D. Hacker, J. Dunlasky \& A. Graesser (Eds.), Handbook of metacognition in education (Cap.1, pp. 26-43). Nueva York: Routledge.

Winograd, P. \& Johnston, P. (1982). Comprehension monitoring and the error detection paradigm. Journal of Reading Behavior, XIV(1), 61-76. http.//dox.doi.org/10.1080/1086296820 9547435

Consejo Nacional de Investigaciones

Cientificas y Técnicas (CONICET)

Facultad de Filosofía y Letras

Universidad de Buenos Aires (UBA)

Ciudad Autónoma de Buenos Aires - República Argentina

Fecha de recepción: 10 de agosto de 2015

Fecha de aceptación: 11 de enero de 2016 